\title{
Early cardiac changes in a rat model of prediabetes: brain natriuretic peptide overexpression seems to be the best marker
}

\author{
Sara Nunes ${ }^{1}$, Edna Soares ${ }^{1}$, João Fernandes ${ }^{2,3}$, Sofia Viana', Eugénia Carvalho ${ }^{4,5}$, Frederico C Pereira ${ }^{1}$
} and Flávio Reis ${ }^{1 *}$

\begin{abstract}
Background: Diabetic cardiomyopathy (DCM) is defined as structural and functional changes in the myocardium due to metabolic and cellular abnormalities induced by diabetes mellitus (DM). The impact of prediabetic conditions on the cardiac tissue remains to be elucidated. The goal of this study was to elucidate whether cardiac dysfunction is already present in a state of prediabetes, in the presence of insulin resistance, and to unravel the underlying mechanisms, in a rat model without obesity and hypertension as confounding factors.
\end{abstract}

Methods: Two groups of 16-week-old Wistar rats were tested during a 9 week protocol: high sucrose (HSu) diet group $(n=7)$ - rats receiving $35 \%$ of sucrose in drinking water vs the vehicle control group $(n=7)$. The animal model was characterized in terms of body weight (BW) and the glycemic, insulinemic and lipidic profiles. The following parameters were assessed to evaluate possible early cardiac alterations and underlying mechanisms: blood pressure, heart rate, heart and left ventricle (LV) trophism indexes, as well as the serum and tissue protein and/or the mRNA expression of markers for fibrosis, hypertrophy, proliferation, apoptosis, angiogenesis, endothelial function, inflammation and oxidative stress.

Results: The HSu-treated rats presented normal fasting plasma glucose (FPG) but impaired glucose tolerance (IGT), accompanied by hyperinsulinemia and insulin resistance $(P<0.01)$, confirming this rat model as prediabetic. Furthermore, although hypertriglyceridemia $(P<0.05)$ was observed, obesity and hypertension were absent. Regarding the impact of the HSu diet on the cardiac tissue, our results indicated that 9 weeks of treatment might be associated with initial cardiac changes, as suggested by the increased LV weight/BW ratio $(P<0.01)$ and a remarkable brain natriuretic peptide (BNP) mRNA overexpression $(P<0.01)$, together with a marked trend for an upregulation of other important mediators of fibrosis, hypertrophy, angiogenesis and endothelial lesions, as well as oxidative stress. The inflammatory and apoptotic markers measured were unchanged.

Conclusions: This animal model of prediabetes/insulin resistance could be an important tool to evaluate the early cardiac impact of dysmetabolism (hyperinsulinemia and impaired glucose tolerance with fasting normoglycemia), without confounding factors such as obesity and hypertension. Left ventricle hypertrophy is already present and brain natriuretic peptide seems to be the best early marker for this condition.

Keywords: Brain natriuretic peptide, Diabetic cardiomyopathy, Fibrosis, Hypertrophy, High-sucrose diet, Prediabetes

\footnotetext{
* Correspondence: freis@fmed.uc.pt

'Laboratory of Pharmacology and Experimental Therapeutics, IBILI, Faculty of

Medicine, University of Coimbra, Coimbra 3000-548, Portugal

Full list of author information is available at the end of the article
} 


\section{Background}

Diabetic cardiomyopathy (DCM) is defined as structural and functional changes in the myocardium, independent of hypertension, chronic artery disease (CAD) or any other known cardiac diseases, which are caused by metabolic and cellular abnormalities induced by diabetes mellitus (DM), ultimately resulting in heart failure (HF) [1-3]. Cardiovascular diseases (CVD) are responsible for three quarters of the deaths among the diabetic population [4] and diabetes accounts for a significant percentage of patients with a diagnosis of HF, which is a major cause of mortality and morbidity in these patients $[5,6]$.

The transition from the early metabolic abnormalities that precedes diabetes, for example impaired fasting glucose (IFG) and impaired glucose tolerance (IGT), to diabetes may take many years; however, current estimates indicate that most individuals with these pre-diabetic states eventually develop DM [7-9]. DCM may be subclinical for a long time, before the appearance of clinical symptoms or signs, complicating its diagnosis and aggravating future complications. However, during the prediabetic state, the risk of cardiovascular events is already increased and myocardial abnormalities might appear prior to the diagnosis of type $2 \mathrm{DM}$. Thus, the earlier identification of cardiac changes in prediabetic/insulin resistant patients could be a better strategy to prevent the evolution to most serious stages of the disease.

One of the most important structural hallmarks of DCM is cardiac hypertrophy $[10,11]$, and this, in turn, is a powerful predictor of CV events [12]. Hyperglycemia has been viewed as the pivotal pathogenetic factor for the development of DCM. In fact, it can cause abnormalities at the cardiac myocyte level, eventually leading to functional and structural abnormalities, including systolic and diastolic dysfunction, as well as cardiac hypertrophy and myocardial fibrosis [13]. However, other factors seem to be involved in the evolution of the disease, including hyperinsulinemia, insulin resistance, oxidative stress, inflammation, endothelial dysfunction and apoptosis $[10,11,14]$. During the last years, the pathological, structural, functional and molecular aspects of the disease have been increasingly investigated, but the issue is far to being elucidated. The knowledge of the cellular and molecular aspects underlying the metabolic disturbances on cardiomyocytes in the prediabetic state should be useful in predicting the structural and functional cardiac consequences. Animal models have been used to study the mechanisms underlying DCM [15-18], but there are limitations. Indeed, the presence of important confounding factors displaying the susceptibility to cardiomyopathy, such as obesity and hypertension, as well as the scarce animal models of prediabetes are major impediments in the progress and understanding of the disease.
Thus, the purpose of this work was to elucidate whether cardiac alteration are already present in a pre diabetic state, and to study its underlying mechanisms, using the high-sucrose ( $\mathrm{HSu}$ ) diet rat model, which is associated with minor metabolic abnormalities and might mimic the human prediabetic state of insulin resistance $[19,20]$, without other complicating factors that could lead to cardiac events.

\section{Material and methods \\ Animals and diet}

Male adult (16 weeks-old) Wistar rats (Charles River Laboratories, Barcelona, Spain), weighting $332.9 \pm 9.0 \mathrm{~g}$ were maintained under controlled temperature $\left(22-23^{\circ} \mathrm{C}\right)$ and light (12:12-h light-dark cycle). After 1 week of acclimatization, animals were randomly divided into two groups ( $\mathrm{n}=7$ each), for a 9-weeks protocol: control - rats continued to receive tap water for drinking; and highsucrose diet $(\mathrm{HSu})$, rats received $35 \%$ sucrose (S0389; Sigma-Aldrich) in the drinking water. All animals were fed standard rat chow, containing $16.1 \%$ of protein, $3.1 \%$ of lipids, $3.9 \%$ of fibers and 5.1\% of minerals (AO4 Panlab, Barcelona, Spain) ad libitum (with exception in the fasting periods). Food and beverage consumption was monitored for both groups throughout the experiment. All experiments were conducted according to the National and European Directives on Animal Care, as well as with ARRIVE guidelines on animal research [21]. The body weight (BW) of each animal was recorded weekly during the experimental period, using an analytical balance (KERN CB 6 K1, Germany).

\section{Blood pressure and heart rate assessment}

Systolic (SBP), diastolic (DBP), mean blood pressure (MBP) and heart rate (HR) values were evaluated in conscious rats using a tail-cuff sphygmomanometer LE 5001 (Letica, Barcelona, Spain) in appropriate contention cages.

\section{Blood and tissue collection and preparation}

At the final time, the rats were subjected to intraperitoneal anesthesia with $50 \mathrm{mg} / \mathrm{kg}$ pentobarbital (Sigma-Aldrich, Portugal) solution and a blood sample was immediately collected by venipuncture from the jugular vein into syringes with Heparin-Lithium (Sarstedt, Monovette ${ }^{\circledR}$ ) for plasma samples and into needles without anticoagulant (for serum samples). The rats were then sacrificed by cervical dislocation, and the heart was immediately removed, placed in ice-cold Krebs' buffer, carefully cleaned of adherent fat and connective tissue, weighted and divided in left and right ventricle. Heart regions were frozen in liquid nitrogen and stored at $-80^{\circ} \mathrm{C}$, for biochemical or gene expression analysis. Before sampling, heart weight (HW) and left ventricle weight (LVW) were measured in order to be 
used as cardiac trophism indexes in relation to body weight: $\mathrm{HW} / \mathrm{BW}$ and LVW/BW.

\section{Metabolic characterization}

Glucose tolerance test (GTT) was performed in fasted rats (6-h) injected intraperitoneally (i.p.) with a glucose bolus of $2 \mathrm{~g} / \mathrm{kg} \mathrm{BW}$. The tail vein blood glucose levels were measured using a portable device (One Touch UltraEasy ${ }^{\circledR}$ glucometer, Lifescan, Johnson and Johnson, Portugal) in samples taken immediately before the bolus and 15, 30, 60, and 120 minutes after. Glycemia was measured in fed conditions.

Insulin tolerance test (ITT) was performed after an i. p. injection of $0.75 \mathrm{U} / \mathrm{kg}$ BW of insulin (I9278, Sigma), in 6-h fasted rats, through monitoring the blood glucose before the injection and 15, 30, 45, 60 and $120 \mathrm{~min}$. after, using the same glucometer. Fasting insulin levels were quantified by using a rat insulin ELISA kit (Mercodia, Uppsala, Sweden). Insulin sensitivity of individual animals was evaluated using the homeostasis model assessment (HOMA) index [22]. The formula used was as follows: [HOMA-IR] = fasting serum glucose $(\mathrm{mg} / \mathrm{dL}) \times$ fasting serum insulin $(\mu \mathrm{U} / \mathrm{mL}) / 22.5$. The values used (insulin and glucose) were obtained after an overnight fasting period.

Serum total cholesterol (TC) and triglycerides (TGs) were analyzed by enzymatic methods using an automatic analyzer (Hitachi 717, Roche Diagnostics). Totalcholesterol reagents and TGs kits were obtained from bioMérieux (Lyon, France).

\section{Serum and heart muscle protein levels and redox status}

Serum levels of transforming growth factor $\beta-1$ (TGF- $\beta 1$ ), vascular endothelial growth factor (VEGF) and interleukin6 (IL-6) were measured through micro-ELISA sandwich assay, using commercial ultrasensitive Quantikine ${ }^{\circledR}$ ELISA kits (R\&D Systems, Minneapolis, USA).

Cardiac expression of the receptor for advanced glycation endproducts (RAGE) and of tumour necrosis factor alpha (TNF- $\alpha$ ) were quantified by western blot. The extracts were obtained from the left ventricle and were homogenized in $1.5 \mathrm{ml}$ of RIPA lysis buffer $(150 \mathrm{mM} \mathrm{NaCl} ; 50 \mathrm{mM}$ Tris $-\mathrm{HCl} \mathrm{pH}=8.0 ; 5 \mathrm{mM}$ EGTA; 1\% Triton X-100; 0.5\% DOC; 0.1\% SDS), supplemented with a protease inhibitor cocktail (1 mM phenylmethylsulfonyl fluoride, $1 \mathrm{mM}$ dithiothreitol, $1 \mu \mathrm{g} /$ $\mathrm{mL}$ chymostatin, $1 \mu \mathrm{g} / \mathrm{mL}$ leupeptin, $1 \mu \mathrm{g} / \mathrm{mL}$ antipain and $5 \mu \mathrm{g} / \mathrm{mL}$ pepstatin A; Sigma-Aldrich) and centrifuged 3 times $\left(15500 \times \mathrm{g}, 15 \mathrm{~min}\right.$., $\left.4^{\circ} \mathrm{C}\right)$. The resulting supernatant fraction (corresponding to total extract) was collected and total protein concentration was determined using BCA assay [23], supernatants were stored at $-80^{\circ} \mathrm{C}$ until further use. Known amounts of total protein $(25 \mu \mathrm{g}$ for RAGE and $90 \mu \mathrm{g}$ for TNF- $\alpha$ ) were loaded and separated by electrophoresis on sodium dodecyl sulfate polyacrylamide gel electrophoresis $(7.5 \%$ and $10 \%$, respectively), transferred to a $0.45 \mu \mathrm{m}$ polyvinylidene difluoride (PVDF) membranes (Immobilon, Millipore, Madrid, Spain) and blocked with $1 \%$ bovine serum albumin (BSA) in phosphate buffer saline with $0.1 \%$ Tween-20 (PBS-T) for $1 \mathrm{~h}$ at room temperature. Membranes were then incubated overnight at $4^{\circ} \mathrm{C}$ with primary antibody (rabbit anti-RAGE, 1:1000; anti-TNF- $\alpha, 1: 600$ ) both from Abcam (Cambridge, UK). The membranes were washed extensively in $0.1 \%$ PBS-T and then incubated for $1 \mathrm{~h}$ at room temperature with alkaline phosphatase conjugated secondary antibodies (goat anti-rabbit, 1:5000, GE Healthcare). Finally, membranes were visualized on Typhoon FLA 900 (GE Healthcare Bio-sciences) imaging system, using an enhanced chemifluorescence detection reagent (ECF, GE Healthcare). To confirm equal protein loading and sample transfer, membranes were reprobed with $\beta$-actin $(1: 5,000$; Sigma-Aldrich) antibodies. Densitometric analyses were performed using the Image Quant 5.0 software. Results were normalized against $\beta$-tubulin, and then expressed as percentage of control.

The thiobarbituric acid reactive-species (TBARs) assay was used to evaluate serum and heart muscle tissue lipid peroxidation, via malondialdehyde (MDA). Samples were analyzed spectrophotometrically at $532 \mathrm{~nm}$ using 1,1,3,3-tetramethoxypropane as an external standard. Serum total antioxidant status (TAS) was measured through ferric reducing antioxidant potential (FRAP) assay, as previously described [24].

\section{RT-qPCR cardiac muscle gene expression}

The heart was isolated and stored in RNA later ${ }^{\text {Twx }}$ solution (Ambion, Austin, TX, USA). In brief, tissue lysates were processed according to the protocol from RNeasy ${ }^{\circledR}$ Mini Kit (Qiagen, Hilden, Germany). RNA integrity (RIN, RNA Integrity Number) was analyzed using 6000 Nano Chip ${ }^{\circledR}$ kit, in Agilent 2100 bioanalyzer (Agilent Technologies, Walbronn, Germany) and 2100 expert software, following manufacturer instructions. The yield from isolation was from 0.5 to $3 \mu \mathrm{g}$; RIN values were 6.0-9.0 and purity (A260/A280) was 1.8-2.0. Reverse transcription and relative quantification of gene expression were performed as previously described [25]. Realtime PCR reactions were performed using the following primer sequences for Bax, Bcl2, brain natriuretic peptide (BNP), connective tissue growth factor (CTGF), intercellular adhesion molecule 1 (ICAM-1), IL-6, inducible nitric oxide synthase (iNOS), Pro-collagen type III, superoxide dismutase (SOD), TGF- $\beta 1$, TNF- $\alpha$, thrombospondin 1 (TSP-1), tribbles 3 (TRB3), vascular cell adhesion molecule 1 (VCAM-1) and VEGF, which were normalized in relation to the expression of beta-actin as an internal control: Bax Forward: 5'-CCAAGAAGC 
TGAGCGAGTGTCTC- ${ }^{\prime}$ and Bax Reverse: ${ }^{\prime}$-AGTTGC CATCAGCAAACATGTCA-3'; Bcl-2 Forward: $5^{\prime}$-GGAG CGTCAACAGGGAGATG-3' and Bcl-2 Reverse: 5'-GA TGCCGGTTCAGGTACTCAG-3'; BNP Forward: 5'-G GGCTGTGACGGGCTGAGGTT-3' and BNP Reverse: 5'-AGTTTGTGCTGGAAGTAAGA-3'; CTGF Forward: 5'-CGTAGACGGTAAAGCAATGG- $3^{\prime}$ and CTGF Reverse: 5'-AGTCAAAGAAGCAGCAAACAC-3'; ICAM-1 Forward: 5'-TTCAACCCGTGCCAGGC-3' and ICAM-1 Reverse: 5'-GTTCGTCTTTCATCCAGTTAGTCT-3'; IL-6 Forward: $5^{\prime}$-ACCACTTCACAAGTCGGAGG- $3^{\prime}$ and IL-6 Reverse: 5'-ACAGTGCATCATCGCTGTTC-3'; iNOS Forward: $5^{\prime}$-CAGAAGCAGAATGTGACCATCAT- $3^{\prime}$ and iNOS Reverse: 5'-CGGAGGGACCAGCCAAATC-3'; Procollagen type III Forward: $5^{\prime}-5^{\prime}$-GGTCACTTTCACT GGTTGACGA-3' and Pro-collagen type III Reverse: 5'-TTGAATATCAAACACGCAAGGC-3'; SOD Forward: 5'-GACA A ACC TGA GCCC TAACGG-3' and SOD Reverse: 5 -CTTCTTGCAAACTATG-3'; TGF- $\beta 1$ Forward: $5^{\prime}$-ATACGCCTGAGTGGCTGTCT- $3^{\prime}$ and TGF- $\beta 1$ Reverse: $5^{\prime}$-TGGGACTGATCCCATTGATT-3'; TNF- $\alpha$ Forward: $5^{\prime}$-CACGCTTTCTGTCTACTGA- $3^{\prime}$ and TNF- $\alpha$ Reverse 5'-GGACTCCGTGATGTCTAAGT-3'; TSP-1 Forward: $5^{\prime}$-CTTTGCTGGTGCCAAGTGTA- ${ }^{\prime}$ and TSP-1 Reverse: $5^{\prime}$-CGACGTCTTTGCACTGGATA-3'; TRB3 Forward: $5^{\prime}$-TGATGCTGTCT GGATGACAA- $3^{\prime}$ and TRB3 Reverse: $5^{\prime}$-GTGAATGGGGACTTTGGTCT-3'; VCAM-1 Forward: $5^{\prime}$-GAA GCCGG TCATGGTCAAGT- $3^{\prime}$ and VCAM-1 Reverse: 5'-GACGGTCACCCTTGAACAG TTC-3'; VEGF Forward: 5'-GAGAATTCGGCCCCAA CCATGAACTTTCTGCT-3' and VEGF Reverse: $5^{\prime}$-G AG CAT G CCCTCCTGCCCGGCTCACCGC-3'; betaactin Forward: $5^{\prime}$-TGTGCTATGTTGCCCTAGACTT C-3' and beta-actin Reverse: 5'-CGGACTCATCGTA CTCCTGCT-3'. Results were analyzed with SDS 2.1 software (Applied Biosystems, Foster City, CA, USA) and relative quantification calculated using the $2-\Delta \Delta \mathrm{Ct}$ method [26].

\section{Statistical analysis}

Results were expressed as means \pm standard errors of the mean (S.E.M.) and \% of the Control, as indicated. Comparisons between groups were analyzed by the unpaired Student's $t$-test, using GraphPad Prism software, Version 5.0. Differences were considered to be significant at $P<0.05$.

\section{Results}

The HSu-diet as a rat model of prediabetes with impaired glucose tolerance and insulin resistance

Beverage consumption was unchanged between groups throughout the experiment. The HSu-treated rats showed a BW profile similar to that of the control animals, during the 9 weeks of treatment (Table 1). Similarly, fasting
Table 1 Body, heart and left ventricle weights, blood pressure, heart rate and lipid profile

\begin{tabular}{lll}
\hline Parameter & $\begin{array}{l}\text { Control group } \\
(\mathbf{n}=\mathbf{7})\end{array}$ & $\begin{array}{l}\text { HSu group } \\
(\mathbf{n}=\mathbf{7})\end{array}$ \\
\hline Body, heart and LV weights & & \\
BW (g) & $390.40 \pm 8.45$ & $397.40 \pm 8.71$ \\
HW (g) & $0.89 \pm 0.09$ & $1.10 \pm 0.01^{*}$ \\
LWW (g) & $0.45 \pm 0.02$ & $0.53 \pm 0.02^{* * *}$ \\
HW/BW (g/kg) & $2.14 \pm 0.23$ & $2.62 \pm 0.07$ \\
LWW/BW (g/kg) & $1.07 \pm 0.02$ & $1.28 \pm 0.04^{* *}$ \\
Blood pressure and heart rate & & \\
SBP (mmHg) & $122.00 \pm 1.79$ & $121.60 \pm 1.10$ \\
DBP (mmHg) & $100.10 \pm 1.55$ & $98.40 \pm 1.32$ \\
MBP (mmHg) & $108.80 \pm 1.27$ & $109.80 \pm 1.23$ \\
HR (beats/min) & $356.70 \pm 1.00$ & $368.90 \pm 8.14$ \\
Lipid profile & & \\
TGs (mg/dl) & $68.14 \pm 9.96$ & $143.10 \pm 23.23^{*}$ \\
TC (mg/dl) & $63.71 \pm 0.94$ & $58.25 \pm 3.44$ \\
\hline
\end{tabular}

Data are expressed as mean \pm SEM, ${ }^{*} \mathrm{P}<0.05$; ${ }^{* *} \mathrm{P}<0.01$; ${ }^{* * \mathrm{P}}<0.001$ versus Control. BW: body weight; DBP: diastolic blood pressure; HR: heart rate; HW: heart weight; LVW: left ventricular weight; MBP: mean blood pressure; SBP: systolic blood pressure; TC: total cholesterol; TGs: triglycerides.

glycemia was unchanged between the two groups (102.90 \pm 6.98 vs $96.71 \pm 4.49 \mathrm{mg} / \mathrm{dl}$ ) (Figure $1 \mathrm{~A}$ ); however, in the fed state, blood glucose levels were significantly elevated in the HSu group compared to Control $(162.90 \pm 26.54$ vs $126.80 \pm 13.56 \mathrm{mg} / \mathrm{dL} ; \mathrm{p}<0.05$ ) (Figure $1 \mathrm{~B}$ ). During a GTT, the HSu-treated rats showed significantly increased blood glucose levels when compared with those of the Control group (215.50 \pm 19.64 vs $159.00 \pm 5.83 \mathrm{mg} / \mathrm{dl} ; \mathrm{p}<$ $0.05), 60 \mathrm{~min}$. after the injection of glucose. This difference persisted until the $120 \mathrm{~min}$., when the blood glucose concentrations returned to basal levels in the Control group, but remained significantly elevated in the $\mathrm{HSu}-$ treated group $(168.80 \pm 10.64$ vs $121.40 \pm 3.69 \mathrm{mg} / \mathrm{dL} ; \mathrm{p}<$ 0.01) (Figure 1C).

Serum fasting insulin concentration in the $\mathrm{HSu}$ treated rats was significantly elevated when compared with the Control animals $(10.83 \pm 1.00$ vs $3.74 \pm 1.84 \mu \mathrm{g} /$ $\mathrm{L} ; \mathrm{p}<0.001$ ) (Figure 1D). A significantly higher value $(\mathrm{p}<0.01)$ of the HOMA-IR index was found in the $\mathrm{HSu}$-treated group, when compared with the Control (Figure 1E). Insulin sensitivity was assessed by the ITT (Figure 1F). The blood glucose levels $120 \mathrm{~min}$. after insulin injection, were significantly elevated in the $\mathrm{HSu}$ treated group than in the Control one $(63.86 \pm 14.78 v \mathrm{~s}$ $37.17 \pm 7.03 \mathrm{mg} / \mathrm{dL} ; \mathrm{p}<0.01)$. TRB3 mRNA expression, in the cardiac muscle tissue, was unchanged in both groups (data not shown). 

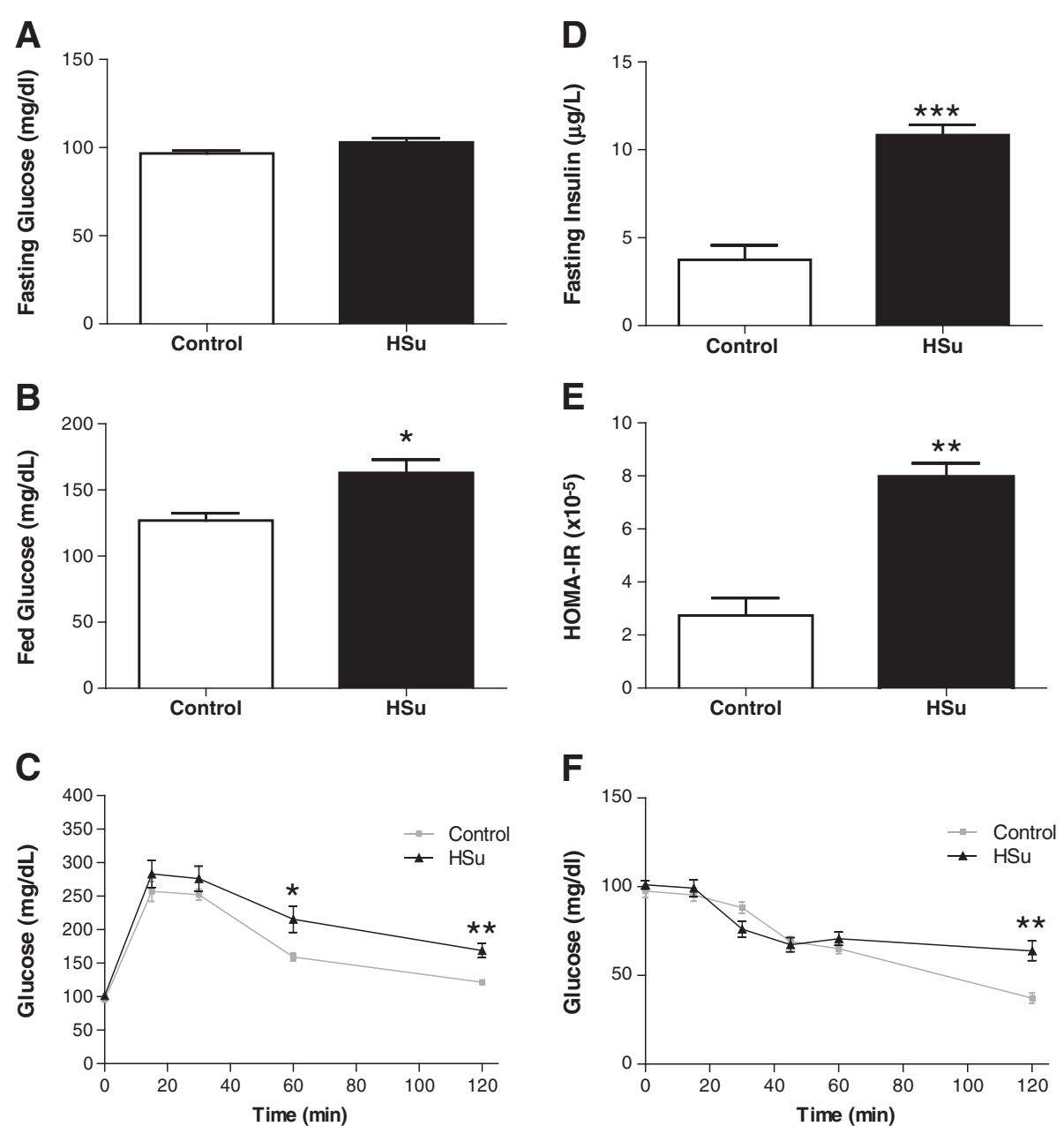

Figure 1 Metabolic characterization of the prediabetic animal model. Glycemic and insulinemic profile: fasting glucose (A), fed glucose (B), glucose tolerance test $(\mathbf{C})$, fasting insulin $(\mathbf{D})$, HOMA-IR index $(\mathbf{E})$ and insulin tolerance test $(\mathbf{F})$, after 9 weeks of treatment. (Results are mean \pm SEM; $n=7$ per group. ${ }^{*} p<0.05 ;{ }^{* *} p<0.01,{ }^{* *} p<0.001$ versus Control group).

HSu-diet induces hypertriglyceridemia without obesity and hypertension

Body weight was unchanged between the groups (Table 1). The Hsu-treated rats presented elevated serum triglyceride contents when compared with the Control, while no significant difference was observed in the total-cholesterol concentration between the two groups (Table 1). No significant differences were found in systolic, diastolic or mean blood pressure, as well as in heart rate, between the two groups (Table 1).

\section{HSu-diet promotes left ventricle hypertrophy with BNP overexpression}

Absolute and relative left ventricle weight (LVW and $\mathrm{LVW} / \mathrm{BW}$ ) was significantly higher in the $\mathrm{HSu}$-treated rats when compared with the Control animals $(\mathrm{p}<0.001$ and $\mathrm{p}$ $<0.01$, respectively) (Table 1 ). To assess the influence of sucrose consumption on proliferation, hypertrophy and fibrosis, the following mediators were evaluated in serum and/or cardiac tissue: BNP, TGF- $\beta 1$, TSP- 1 , pro-collagen III and CTGF. No significant changes were found concerning the cardiac mRNA expression of TGF- $\beta 1$, TSP1 , pro-collagen III and CTGF, despite a trend to higher values in the $\mathrm{HSu}$ animals (Figure 2A). Serum TGF- $\beta 1$ levels were also identical between groups, despite a trend to higher values in the $\mathrm{HSu}$-treated rats (Figure $3 \mathrm{~A}$ ). However, a significant $>5$ fold increase $(\mathrm{p}<0.01)$ in BNP mRNA expression was present in the HSu-treated animals when compared with Control (Figure 2A).

HSu-diet did not change cardiac markers of apoptosis, inflammation, angiogenesis and endothelial lesions

No changes were found in cardiac muscle for apoptotic markers, Bax and Bcl-2 mRNA expression, between the two groups. iNOS, ICAM-1 and VCAM-1 gene expression was also identical for both groups (Figure 2B). A trend to increased cardiac VEGF mRNA expression was found in the HSu-treated animals, with a significantly 


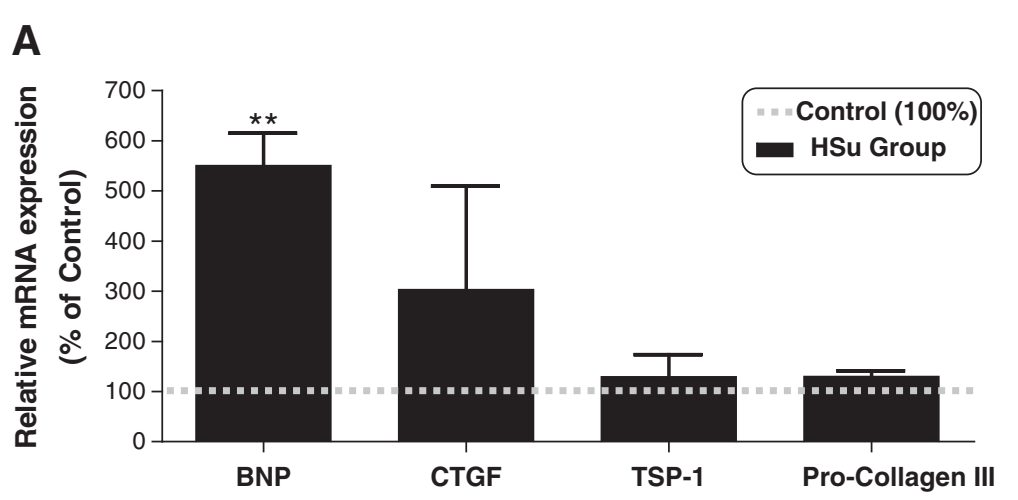

B

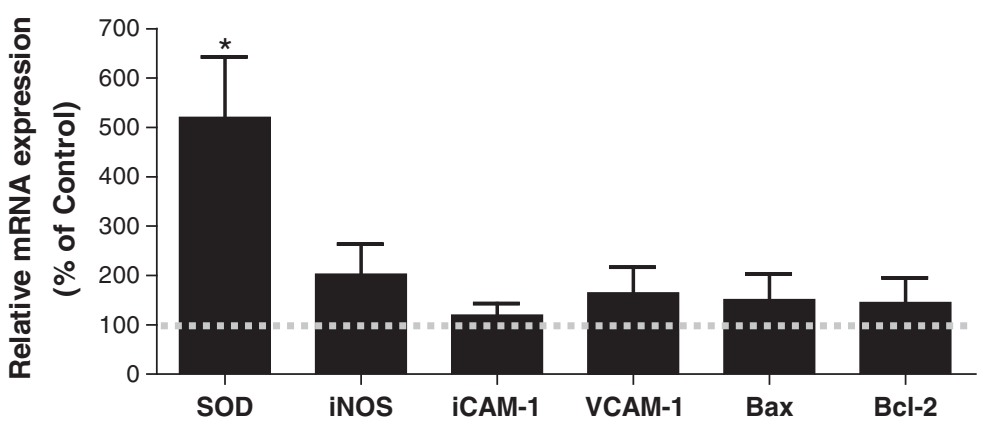

Figure 2 Cardiac gene expression profile. mRNA expression of mediators of proliferation, fibrosis and hypertrophy (A: BNP, CTGF, TSP-1 and Pro-collagen III) and of endothelial lesion and apoptosis (B: SOD, iNOS, ICAM-1, VCAM-1, Bax and BCI2), after 9 weeks of treatment. (Results are means \pm SEM of relative expression in relation to the control; $n=7$ per group. ${ }^{*} p<0.05$ and ${ }^{* *} p<0.01$ versus Control group).

higher $(\mathrm{p}<0.05)$ serum VEGF concentration, when compared with the control rats (Figure $3 \mathrm{~B}$ and $\mathrm{D}$ ). Both cardiac IL-6 mRNA expression and circulating levels were unchanged between groups, (Figure $3 \mathrm{C}$ and D). TNF- $\alpha$ mRNA expression was also identical between groups (data not shown).

\section{Effect of HSu treatment on redox status markers}

Both serum and heart tissue lipid peroxidation showed a trend for an increase, although the levels did not achieve statistical significance (Figure 4A and B). However, serum TAS was significantly increased $(\mathrm{p}<0.05)$ in the animals treated with sucrose (Figure $4 \mathrm{C}$ ), which was accompanied by a significant increase $(\mathrm{p}<0.05)$ in SOD mRNA overexpression and a significant reduction in RAGE levels in cardiac muscle $(\mathrm{p}<0.01)$ (Figure $2 \mathrm{~B})$.

\section{Discussion}

The high-sucrose diet rat as a model of prediabetes

Many reports have demonstrated that consumption of high-sucrose diet induces impaired glucose tolerance and hyperinsulinemia, and decreases insulin sensitivity [27-29]. However, data from models of prediabetes, namely the HSu-treated rat, are scarce $[15,16]$. Our study demonstrates no differences on glycemia in the fasting state, but a significant increase in the fed (postprandial) state in $\mathrm{HSu}$-treated rats, together with impaired glucose tolerance, hyperinsulinemia and insulin resistance. This is line with previous studies having demonstrated that sucrose consumption produces insulin resistance in rats $[19,20]$, which is characterized by fasting normoglycemia $[30,31]$. Thus, HSu-evoked impaired glucose tolerance as well as hyperinsulinemia might be triggered by peripheral insulin resistance. In fact, T2DM is recognized as a progressive disorder, which is associated with diminishing pancreatic function over time. However, at an early stage, as it may occur after 9 weeks of high sucrose intake, the presence of insulin resistance must be compensated by hyperinsulinemia to maintain normoglycemia. This is the case in individuals who develop diabetes, a progressive loss of the insulin secretory capacity of $\beta$-cells can appear much before the clinical diagnosis [32]. Furthermore, TRB3 (a member of mammalian tribbles homologs protein family), has been recently considered to be a mediator of insulin resistance in DCM [33], explaining our interest in its evaluation in this model. However, our result showed no differences in TRB3 mRNA expression between the groups, suggesting that it might not be impaired in the sucroseinduced prediabetic state.

The present study demonstrated that sucrose intake induced no significant changes on body weight and total 

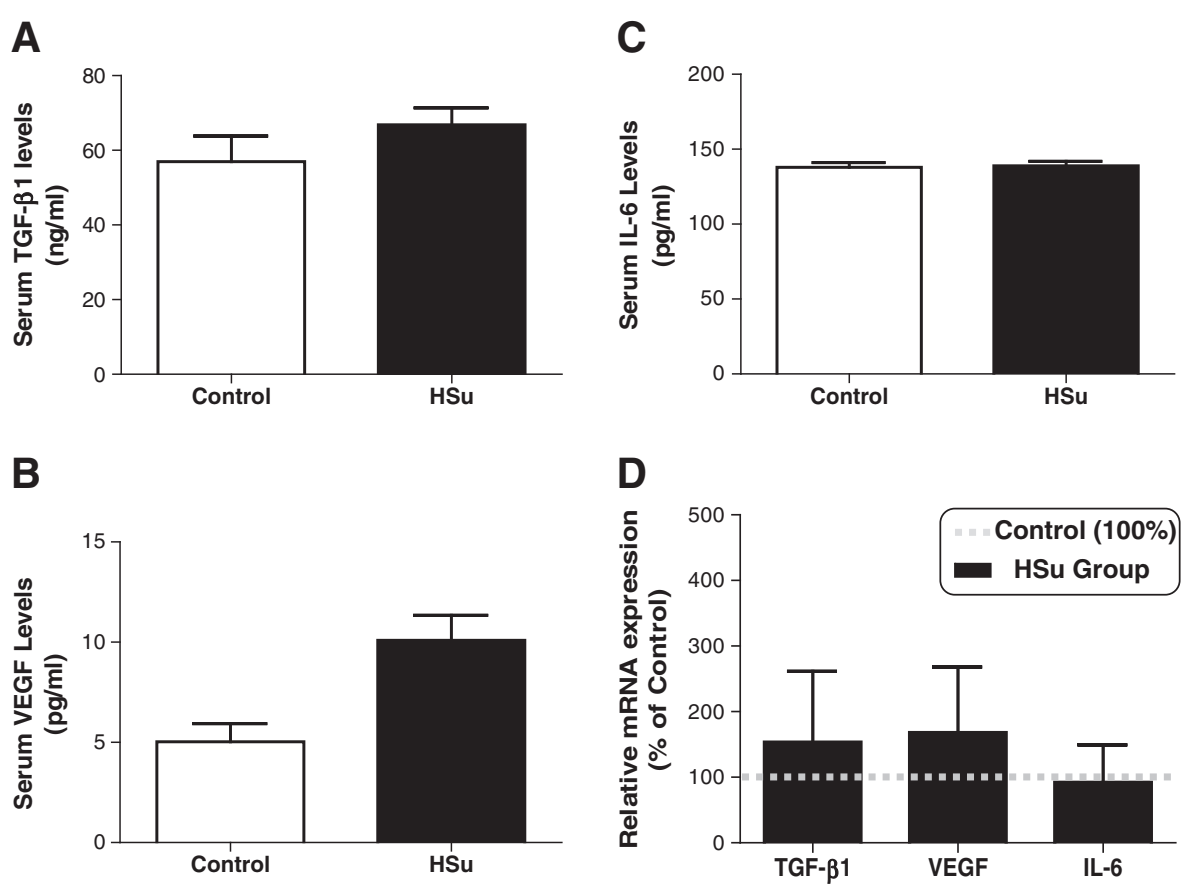

Figure 3 Serum and cardiac tissue protein/gene expression profile. Serum TGF- $\beta 1$ (A), VEGF (B) and IL-6 (C) concentrations and cardiac mRNA expression of TGF- $\beta 1, V E G F$ and IL-6 (D), after 9 weeks of treatment. (Results are means \pm SEM; relative expression in relation to the control for mRNA levels; $n=7$ per group. ${ }^{*} p<0.05$ versus Control group).
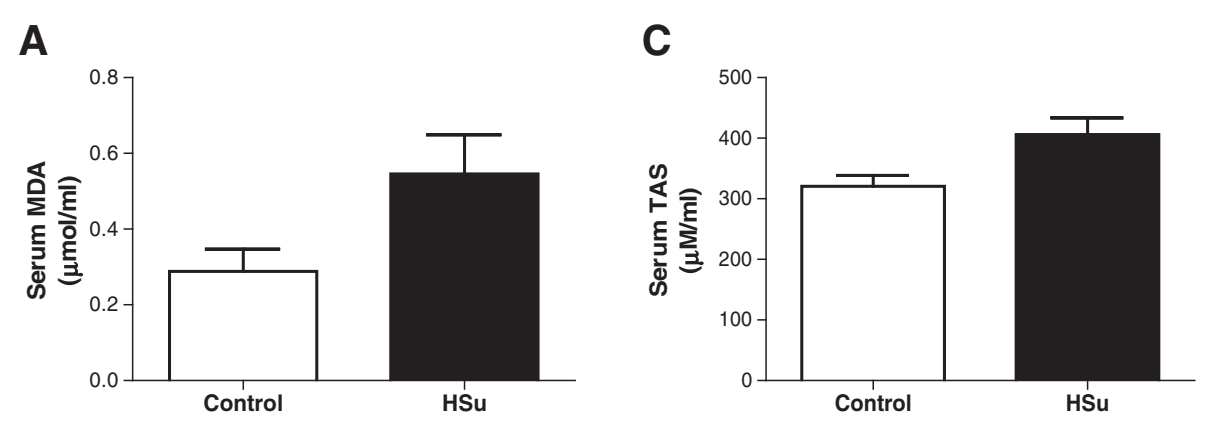

B

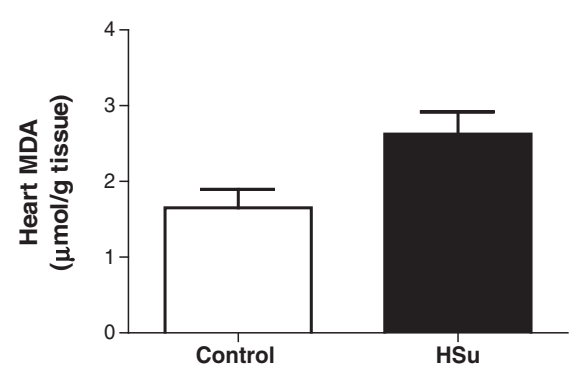

D

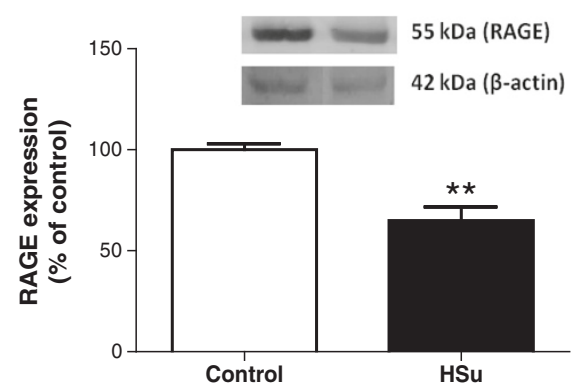

Figure 4 Serum and cardiac tissue redox status markers. Serum (A) and heart (B) lipid peroxidation, serum total antioxidant status (C) and cardiac RAGE mRNA expression (D), after 9 weeks of treatment. (Results are means \pm SEM; relative expression in relation to the control for $m R N A$ levels; $n=7$ per group. ${ }^{*} p<0.05$ and ${ }^{* *} p<0.01$ versus Control group). 
cholesterol levels, suggesting that insulin resistance produced in this model is, at least initially, independent of the overt obesity, which is consistent with a previous study [28]. However, elevated serum TGs contents were observed in the HSu-treated rats consistent with other reports [28,34]. This may be a result of de novo lipogenesis, the enhanced rate of hepatic TGs synthesis and the decrease in peripheral clearance [35]. In addition, no significant differences in blood pressure and heart rate were observed between $\mathrm{HSu}$-treated rats and the control animals, indicating that 9 weeks of $35 \%$ sucrose treatment was unable to cause a significant impact on these parameters. The data is in agreement with a previous work, which failed to show hypertension in high-sucrose diet fed rats [20].

The mechanisms underlying the development of diabetic cardiomyopathy have been widely discussed and seem to involve complex and multifactorial influences. Indeed, as in diabetes, hyperglycemia, hyperinsulinemia and hyperlipidemia might be the initial triggers of significant cellular and molecular changes ultimately leading to cardiac functional and structural impairment, key features of the systolic and diastolic dysfunction associated with diabetic cardiomyopathy. Oxidative stress, endothelial dysfunction, inflammation and apoptosis, are important triggers of cardiac fibrosis and hypertrophy, which are viewed as major structural changes associated with cardiac dysfunction inherent to DCM [15-18]. The existence of earlier cardiac changes in prediabetes has been suggested, but the human studies, and even the animal models, are usually limited because of the coexistence of other perturbations. Our data is consistent with a model of prediabetes/insulin resistance, characterized by fasting normoglycemia, impaired glucose tolerance, hyperinsulinemia, insulin resistance, together with hypertriglyceridemia, important features to regarding their direct impact on cardiac tissue, independently of other confounding factors, such as hypertension and obesity.

\section{Early markers of cardiac hypertrophy and/or fibrosis: focus on BNP overexpression}

Cardiomyocyte hypertrophy, as well as subsequent apoptosis and myocardial fibrosis, are structural hallmarks of DCM [36,37]. Previous studies have shown that cardiomyocyte hypertrophy in diabetes is regulated at the transcriptional level $[38,39]$. Thus, it is important to examine molecular biomarkers of the putative cardiac alterations. In this study, we evaluated cardiac mRNA levels of TGF- $\beta 1$, TSP- 1 , pro-collagen III, CTGF and BNP, which have been associated with cardiac hypertrophy and/or fibrosis. Indeed, TGF- $\beta 1$ plays a role in cell growth, differentiation, apoptosis, inflammatory processes and gene expression [40] and has consistent implications in DCM due to pro-fibrotic properties, including the expression of many matrix proteins, such as collagens. In the hearts of diabetic patients, collagen remodeling occurs in the perimysium and perivascular region, mainly as a result of an increased accumulation of type III collagen [41], which has been related to leftventricular diastolic and systolic dysfunction in DCM $[42,43]$. Additionally, TSP-1 is an adhesive glycoprotein that influences cellular phenotype and the structure of the extracellular matrix [44]. It has been reported that high glucose levels stimulates TGF- $\beta 1$ bioactivation by fibroblasts through up-regulation of TSP-1 [45]. CTGF is another potent pro-fibrotic protein that plays important roles in tissue and organ fibrosis and it is increasingly being implicated in structural and functional abnormalities in the diabetic heart $[46,47]$. In addition, $\mathrm{BNP}$, a peptide hormone released from the cardiac ventricles in response to pressure and volume overload, is among the most relevant molecular markers of cardiac hypertrophy [48]. In our study, no significant differences were found in cardiac gene expression of TGF- $\beta 1$, procollagen III, TSP-1 and CTGF between the groups, despite a trend to increased values in the $\mathrm{HSu}$ animals for all mediators. However, BNP gene expression was significantly higher in animals under sucrose treatment, which is in agreement with the LV hypertrophy, viewed by the relative increase in LV weight. Thus, collectively, this data indicate that a high sucrose diet can promote cardiac trophism in a prediabetic state, with BNP the earlier biomarker of those alterations, in agreement with a recent study suggesting BNP as a useful marker for screening preclinical ventricular diastolic dysfunction in patients prone to develop cardiovascular complications, such as uncontrolled diabetics [49]. Cardiac hypertrophy and fibrosis are usually associated with apoptosis [50]. In this study, no significant changes in Bax and Bcl-2 cardiac gene expression were found. Whether these are or not related with the anti-apoptotic effect of insulin in the absence of hyperglycemia, deserves further evaluation [51].

\section{Markers of endothelial dysfunction and angiogenesis}

Endothelial dysfunction is one of the mechanisms involved in the pathogenesis of DCM. Inducible NOS is an enzyme with recognized implications in ROS production, particularly under inflammatory conditions, and it is also involved in insulin resistance [52]. Activation of iNOS contributes to the pathogenesis of CVD, probably because of excessive release of $\mathrm{NO}$ and generation of ROS. Ropelle et al. (2013) recently demonstrated that iNOS overexpression induced by aging is associated with increased S-nitrosation of major proteins involved in insulin signalling, thus reducing insulin sensitivity in skeletal muscle, which is prevented in aged iNOS-null mice 
[53]. In addition, iNOS deficiency protects from high-fat diet-induced insulin resistance in the obese Zucker rat model [54]. In our model, no significant changes in cardiac iNOS gene levels were found, despite a trend for higher values in the insulin resistant animals. Lee et al. (2009) suggest that upregulation of iNOS may be a protective mechanism against excessive contraction, abnormal signalling resulting from oxidative stress and enhanced inflammation in the diabetic vasculature [55]. Whether this hypothetic protection is present or not in our model, needs additional investigation. Endothelial dysfunction was also assessed by the expression of adhesion molecules, such as ICAM-1 and VCAM-1, which have been associated with the development of CVD in diabetic patients $[56,57]$. In our model, however, no differences in cardiac ICAM-1 and VCAM-1 gene expression were found, suggesting they could be later markers of these changes. Another mediator of vascular changes found in diabetic patients is VEGF, promoting angiogenesis and increasing vascular permeability $[58,59]$. We observed a trend for increased levels in cardiac tissue VEGF in HSu-treated rats, which was accompanied by increased serum protein levels, suggesting its direct involvement.

\section{Markers of inflammation and redox status}

Elevated inflammatory cytokines have been found in circulation and in the diabetic hearts of T2DM patients, contributing to heart failure [60-62]. Cardiac overexpression of TNF- $\alpha$ has been associated with cardiac hypertrophy and fibrosis, as well with left ventricular dysfunction $[63,64]$ and IL-6 has been also described as an inducer of myocardial damage by promoting LV dysfunction and cardiac hypertrophy under acute myocardial infarction [65]. In our study, however, no significant changes were found in either the serum or cardiac levels for IL- 6 and cardiac TNF- $\alpha$, suggesting that inflammation is not the key factor of the early changes related to cardiomyopathy.

In diabetes, oxidative stress has been recognized as an important player in the mechanism underlying DCM. However, in a state of insulin resistance without hyperglycemia, as it occurs in the early stages of diabetes (prediabetes), the contribution of ROS and consequent oxidative stress to the heart remains to be elucidated. In our model, the absence of markers of increased inflammation was accompanied by slight changes in the redox status measured. We found a trend for increased MDA levels (a marker of lipid peroxidation) in both the serum and heart tissue of HSu-treated rats. However, a significantly increased in serum TAS (a measure of total antioxidant capacity) was observed in the HSu rats, accompanied by an overexpression of the cardiac SOD gene, as well as a reduced expression of RAGE. These might collectively be viewed as compensatory actions against the putative increase in ROS. Furthermore, our model presented hyperinsulinemia, which was still able to normalize blood glucose levels, despite the impaired glucose tolerance. Thus, the putative generation of ROS, as suggested by the small increase in serum and heart lipid peroxidation, seems to be compensated by additional generation of antioxidants, observed by the significantly increased serum TAS and cardiac gene expression of SOD. Furthermore, the observed underexpression of RAGE in cardiac tissue might be also viewed as a compensatory mechanism, protecting the cardiac tissue. Indeed, we cannot exclude the possibility that longer exposure times of sucrose may lead to reduction of these antioxidant defence mechanisms and, consequently, to toxic effects induced by the oxidative stress aggravating cardiac function.

Hyperglycemia has been indicated as one of the major players in DCM, due to activation of several mechanisms, including promotion of oxidative stress. In our model, the modifications present at an early stage (hyperinsulinemia, insulin resistance, fasting normoglycemia) before over diabetes (defined as prediabetes), might be eventually linked with the impact of impaired glucose tolerance (fed, or postprandial, hyperglycemia). Additional studies are needed to better understand the underlying molecular and cellular mechanisms responsible for the effects of $\mathrm{HSu}$ in prediabetes, as well as to elucidate whether hyperglycemia is, or not, the major driving force for the overt cardiac changes underlying cardiomyopathy.

\section{Conclusion}

This animal model of prediabetes/insulin resistance, induced by high sucrose consumption during 9 weeks, could be an important tool to evaluate the cardiac impact of dysmetabolism (mainly hyperinsulinemia and impaired glucose tolerance, with fasting normoglycemia), without confounding factors such as obesity and hypertension. Left ventricle hypertrophy is the main characteristic and BNP overexpression seems to be the best early marker.

\footnotetext{
Abbreviations

BNP: Brain natriuretic peptide; BSA: Bovine serum albumin; BW: Body weight; CAD: Chronic artery disease; CTGF: Connective tissue growth factor; CVD: Cardiovascular diseases; DBP: Diastolic blood pressure; DCM: Diabetic cardiomyopathy; DM: Diabetes mellitus; GTT: Glucose tolerance test; HF: Heart failure; HOMA-IR: Homeostasis model assessment-Insulin Resistance; HR: Heart rate; HSu: High sucrose; HW: Heart weight; ICAM-1: Intercellular adhesion molecule-1; IFG: Impaired fasting glucose; IGT: Impaired glucose tolerance; IL-6: Interleukin-6; iNOS: Inducible nitric oxide synthase; ITT: Insulin tolerance test; FPG: Fasting plasma glucose; FRAP: Ferric reducing antioxidant potential; LV: Left ventricle; LWW: Left ventricle weight;

MDA: Malondialdehyde; MBP: Mean blood pressure; RAGE: Receptor of advanced glycation endproducts; SBP: Systolic blood pressure;

SOD: Superoxide dismutase; TAS: Total antioxidant status;

TBARs: Thiobarbituric acid reactive-species, TC, Total cholesterol; TGF-

$\beta 1$ : Transforming growth factor $\beta-1 ;$ TGs: Triglycerides; TNF-a: Tumour
} 
necrosis factor alpha; TRB3: Tribbles 3; TSP-1: Thrombospondin-1; VCAM1: Vascular cell adhesion molecule-1; VEGF: Vascular endothelial growth factor.

\section{Competing interest}

The authors report no competing interest.

\section{Authors' contributions}

SN, EC, FCP and FR conceived and designed the study protocol. SN, ES, JF, SV and EC performed the analytical assays. SN, EC, FCP and FR prepared the manuscript. All authors have read and approved the manuscript.

\section{Acknowledgement}

This study was supported by the Portuguese Foundation for Science and Technology, through Strategic Project PEst-C/SAU/UI3282/2011, PTDC/SAUOSM/104124/2008 and COMPETE, as well as by a Grant of the Group fo Fundamental and Translational Investigation (GIFT) of the Portuguese Society of Diabetology (SPD).

\section{Author details}

'Laboratory of Pharmacology and Experimental Therapeutics, IBILI, Faculty of Medicine, University of Coimbra, Coimbra 3000-548, Portugal. ${ }^{2}$ Biochemistry Department, Faculty of Pharmacy, University of Porto, Porto, Portugal. ${ }^{3}$ Institute for Molecular and Cellular Biology, University of Porto, Porto, Portugal. ${ }^{4}$ Center for Neuroscience and Cell Biology, University of Coimbra, Coimbra, Portugal. ${ }^{5}$ The Portuguese Diabetes Association (APDP), Lisbon, Portugal.

Received: 11 February 2013 Accepted: 4 March 2013

Published: 7 March 2013

\section{References}

1. Tarquini R, Lazzeri C, Pala L, Rotella CM, Gensini GF: The diabetic cardiomyopathy. Acta Diabetol 2011, 48(3):173-181.

2. Fang ZY, Prins JB, Marwick TH: Diabetic cardiomyopathy: evidence, mechanisms, and therapeutic implications. Endocr Rev 2004, 25(4):543-567.

3. Tziakas DN, Chalikias GK, Kaski JC: Epidemiology of the diabetic heart. Coron Artery Dis 2005, 16(Suppl 1):S3-S10.

4. Khavandi K, Khavandi A, Asghar O, Greenstein A, Withers S, Heagerty AM, Malik RA: Diabetic cardiomyopathy- a distinct disease? Best Pract Res Clin Endocrinol Metab 2009, 23:347-360

5. Nieminen MS, Brutsaert D, Dickstein K, Drexler H, Follath F, Harjola VP, Hochadel M, Komajda M, Lassus J, Lopez-Sendon JL, Ponikowski P, Tavazzi L, EuroHeart Survey Investigators; Heart Failure Association, European Society of Cardiology: EuroHeart Failure Survey II (EHFS II): a survey on hospitalized acute heart failure patients: description of population. Eur Heart J 2006, 27:2725-2736.

6. Cohen-Solal A, Beauvais F, Loqeart D: Heart failure and diabetes mellitus: epidemiology and management of an alarming association. J Card Fail 2008, 14:615-625.

7. Wang H, Shara NM, Calhoun D, Umans JG, Lee ET, Howard BV: Incidence rates and predictors of diabetes in those with prediabetes: the strong heart study. Diabetes Metab Res Rev 2010, 26(5):378-385.

8. Nguyen QM, Srinivasan SR, Xu JH, Chen W, Berenson GS: Fasting plasma glucose levels within the normoglycemic range in childhood as a predictor of prediabetes and type 2 diabetes in adulthood: the Bogalusa Heart Study. Arch Pediatr Adolesc Med 2010, 164(2):124-128.

9. Colagiuri S: Epidemiology of prediabetes. Med Clin North Am 2011, 95(2):299-307.

10. Voulgari C, Papadogiannis D, Tentolouris N: Diabetic cardiomyopathy: from the pathophysiology of the cardiac myocytes to current diagnosis and management strategies. Vasc Health Risk Manag 2010, 6:883-903.

11. Boudina S, Abel ED: Diabetic cardiomyopathy, causes and effects. Rev Endocr Metab Disord 2010, 11(1):31-39.

12. Levy D, Garrison RJ, Savage DD, Kannel WB, Castelli WP: Prognostic implications of echocardiographically determined left ventricular mass in the Framingham Heart Study. N Engl J Med 1990, 322:1561-1566.

13. Murarka S, Movahed MR: Diabetic cardiomyopathy. J Card Fail 2010, 16(12):971-979.
14. Nunes $S$, Soares $E$, Pereira FC, Reis F: The role of inflammation in diabetic cardiomyopathy. Int J Infereron Cytokine Mediator Res 2012, 4:59-73.

15. Fredersdorf S, Thumann C, Zimmermann WH, Vetter R, Graf T, Luchner A, Riegger GA, Schunkert H, Eschenhagen T, Weil J: Increased myocardial SERCA expression in early type 2 diabetes mellitus is insulin dependent: In vivo and in vitro data. Cardiovasc Diabetol 2012, 11:57.

16. Hou L, Lian K, Yao M, Shi Y, Lu X, Fang L, He T, Jiang L: Reduction of n-3 PUFAs, specifically DHA and EPA, and enhancement of peroxisomal betaoxidation in type 2 diabetic rat heart. Cardiovasc Diabetol 2012, 11:126.

17. Aragno M, Mastrocola R, Ghé C, Arnoletti E, Bassino E, Alloatti G, Muccioli G: Obestatin induced recovery of myocardial dysfunction in type 1 diabetic rats: underlying mechanisms. Cardiovasc Diabetol 2012, 11:129.

18. Bugger $\mathrm{H}$, Abel ED: Rodent models of diabetic cardiomyopathy. Dis Model Mech 2009, 2(9-10):454-466.

19. Pagliassotti MJ, Prach PA, Koppenhafer TA, Pan DA: Changes in insulin action, triglycerides, and lipid composition during sucrose feeding in rats. Am J Physiol 1996, 271:R1319-R1326.

20. Santuré $M$, Pitre $M$, Marette $A$, Deshaies $Y$, Lemieux C, Larivière $R$, Nadeau $A$, Bachelard $\mathrm{H}$ : Induction of insulin resistance by high-sucrose feeding does not raise mean arterial blood pressure but impairs haemodynamic responses to insulin in rats. Br J Pharmacol 2002, 137(2):185-196.

21. Kilkenny C, Browne WJ, Cuthill IC, Emerson M, Altman DG: Improving bioscience research reporting: the ARRIVE guidelines for reporting animal research. PLoS Biol 2010, 8:e1000412.

22. Matthews DR, Hosker JP, Rudenski AS, Naylor BA, Treacher DF, Turner RC Homeostasis model assessment: insulin resistance and $\beta$-cell function from fasting plasma glucose and insulin concentration in man. Diabetologia 1985, 28:412-419.

23. Smith $P$, Krohn R, Hermanson G, Mallia A, Gartner F, Provenzano M, Fujimoto E, Goeke N, Olson B, Klenk D: Measurement of protein using bicinchoninic acid. Anal Biochem 1985, 150(1):76-85.

24. Piloto N, Teixeira HM, Teixeira-Lemos E, Parada B, Garrido P, Sereno J, Pinto R, Carvalho L, Costa E, Belo L, Santos-Silva A, Teixeira F, Reis F:

Erythropoietin promotes deleterious cardiovascular effects and mortality risk in a rat model of chronic sports doping. Cardiovasc Toxicol 2009, 9:201-210.

25. Teixeira M, Rodrigues-Santos P, Garrido P, Costa E, Parada B, Sereno J, Alves R, Belo L, Teixeira F, Santos-Silva A, Reis F: Cardiac antiapoptotic and proproliferative effect of recombinant human erythropoietin in a moderate stage of chronic renal failure in the rat. J Pharm Bioallied SCi 2012, 4:76-83.

26. Livak KJ, Schmittgen TD: Analysis of relative gene expression data using real -time quanti-tative PCR and the $2^{-\Delta \Delta C T}$. Method Methods 2001, 25:402-408.

27. Ribeiro RT, Lautt WW, Legare DJ, Macedo MP: Insulin resistance induced by sucrose feeding in rats is due to an impairment of the hepatic parasympathetic nerves. Diabetologia 2005, 48(5):976-983

28. Sumiyoshi M, Sakanaka M, Kimura Y: Chronic intake of high-fat and highsucrose diets differentially affects glucose intolerance in mice. J Nutr 2006, 136(3):582-587.

29. Ghafoorunissa, Ibrahim A, Natarajan S: Substituting dietary linoleic acid with alpha-linolenic acid improves insulin sensitivity in sucrose fed rats. Biochim Biophys Acta 2005, 1733(1):67-75.

30. Thresher JS, Podolin DA, Wei Y, Mazzeo RS, Pagliassotti MJ: Comparison of the effects of sucrose and fructose on insulin action and glucose tolerance. Am J Physiol Regul Integr Comp Physiol 2000, 279(4):R1334-R1340.

31. Wright D, Hansen R, Mondon C, Reaven G: Sucrose-induced insulin resistance in the rat: modulation by exercise and diet. Am J Clin Nutr 1983, 38:879-883.

32. Cefalu WT: Animal models of type 2 diabetes: clinical presentation and pathophysiological relevance to the human condition. ILAR J 2006, 47(3):186-198

33. Ti Y, Xie GL, Wang ZH, Bi XL, Ding WY, Wang J, Jiang GH, Bu PL, Zhang Y, Zhong $M$, Zhang W: TRB3 gene silencing alleviates diabetic cardiomyopathy in a type 2 diabetic rat model. Diabetes 2011, 60(11):2963-2974.

34. Kanazawa M, Xue CY, Kageyama H, Suzuki E, Ito R, Namba Y, Osaka T, Kimura S, Inoue S: Effects of a high-sucrose diet on body weight, plasma triglycerides, and stress tolerance. Nutr Rev 2003, 61:S27-S33.

35. Suzuki $\mathrm{T}$, Hara $\mathrm{H}$ : Ingestion of guar gum hydrolysate, a soluble and fermentable nondigestible saccharide, improves glucose intolerance and prevents hypertriglyceridemia in rats fed fructose. J Nutr 2004, 134:1942-1947. 
36. Chen S, Evans T, Mukherjee K, Karmazyn M, Chakrabarti S: Diabetes-induced myocardial structural changes: role of endothelin-1 and its receptors. J Mol Cell Cardiol 2000, 32:1621-1629.

37. Li CJ, LV L, Li H, Yu DM: Cardiac fibrosis and dysfunction in experimental diabetic cardiomyopathy are ameliorated by alpha-lipoic acid. Cardiovasc Diabetol 2012, 11:73

38. Chen S, Khan ZA, Cukiernik M, Chakrabarti S: Differential activation of NF-_B and AP-1 in increased fibronectin synthesis in target organs of diabetic complications. Am J Physiol Endocrinol Metab 2003, 284:E1089-E1097.

39. Feng B, Chen S, Chiu J, George B, Chakrabarti S: Regulation of cardiomyocyte hypertrophy in diabetes at the transcriptional level. Am J Physiol Endocrinol Metab 2008, 294(6):E1119-E1126.

40. Bujak M, Frangogiannis N: The role of TGF-beta signaling in myocardial infarction and cardiac remodeling. Cardiovasc Res 2007, 74(2):184-195.

41. Shimizu M, Umeda K, Sugihara N, Yoshio H, Ino H, Takeda R, Okada Y, Nakanishi I: Collagen remodelling in myocardial of patients with diabetes. J Clin Pathol 1993, 46(1):32-36.

42. Mizushige K, Yao L, Noma T, Kiyomoto H, Yu Y, Hosomi N, Ohmori K, Matsuo $\mathrm{H}$ : Alteration in left ventricular diastolic filling and accumulation of myocardial collagen at insulin-resistant prediabetic stage of a type II diabetic rat model. Circulation 2000, 101(8):899-907.

43. Westermann D, Rutschow $\mathrm{S}$, Jager $\mathrm{S}$, Linderer A, Anker S, Riad A, Unger T, Schultheiss HP, Pauschinger M, Tschöpe C: Contributions of inflammation and cardiac matrix metallopro-teinase activity to cardiac failure in diabetic cardiomyopathy: the role of angiotensin type 1 receptor antagonism. Diabetes 2007, 56(3):641-646.

44. Lawler J: Thrombospondin-1 as an endogenous inhibitor of angiogenesis and tumor growth. J Cell Mol Med 2002, 6(1):1-12.

45. Yung S, Lee CYY, Zhang Q, Lau SK, Tsang RCW, Chan TM: Elevated glucose induction of thrombospondin-1 up-regulates fibronectin synthesis in proximal renal tubular epithelial cells through TGF-b1 dependent and TGF-b1 independent pathways. Nephrol Dial Transplant 2006, 21:1504-1513

46. Leask A, Holmes A, Abraham DJ: Connective tissue growth factor: a new and important player in the pathogenesis of fibrosis. Curr Reumatol Rep 2002, 4:136-142.

47. Way KJ, Isshiki K, Suzuma K, Yokota T, Zvagelsky D, Schoen FJ, Sandusky GE, Pechous PA, Vlahos CJ, Wakasaki H, King GL: Expression of connective tissue growth factor is increased in injured myocardium associated with protein kinase $\mathrm{C}$ beta2 activation and diabetes. Diabetes 2002, 51:2709-2718

48. Vanderheyden M, Bartunek J, Goethals M: Brain and other natriuretic peptides: molecular aspects. Eur J Heart Fail 2004, 6(3):261-268.

49. Romano S, Di Mauro M, Fratini S, Guarracini L, Guarracini F, Poccia G, Penco M: Early diagnosis of left ventricular diastolic dysfunction in diabetic patients: a possible role for natriuretic peptides. Cardiovasc Diabetol 2010, 9:89.

50. Reinking BE, Wedemeyer EW, Weiss RM, Segar JL, Scholz TD Cardiomyopathy in offspring of diabetic rats is associated with activation of the MAPK and apoptotic pathways. Cardiovasc Diabeto/ 2009, 8:43.

51. Aikawa R, Nawano M, Gu Y, Katagiri H, Asano T, Zhu W, Nagai R, Komuro I: Insulin prevents cardiomyocytes from oxidative stress-induced apoptosis through activation of PI3 kinase/Akt. Circulation 2000, 102:2873-2879.

52. Soskić SS, Branislava DD, Sudar EM, Obradović MM, Nikolić DM, Djordjevic JD, Radak DJ, Mikhailidis DP, Isenović ER: Regulation of Inducible Nitric Oxide Synthase (iNOS) and its potential role in insulin resistance, diabetes and heart failure. Open Cardiovasc Med J 2011, 5:153-163.

53. Ropelle ER, Pauli JR, Cintra DE, da Silva AS, De Souza CT, Guadagnini D, Carvalho BM, Caricilli AM, Katashima CK, Carvalho-Filho MA, Hirabara S, Cur R, Velloso LA, Saad MJ, Carvalheira JB: Targeted disruption of inducible nitric oxide synthase protects against aging, s-nitrosation, and insulin resistance in muscle of male mice. Diabetes 2013, 62:466-470.

54. Zhou YT, Grayburn P, Karim A, Shimabukuro M, Higa M, Baetens D, Orci L, Unger RH: Lipotoxic heart disease in obese rats: implications for human obesity. Proc Natl Acad Sci USA 2000, 97:1784-1789.

55. Lee JH, Palaia T, Ragolia L: Impaired insulin-mediated vasorelaxation in diabetic Goto-Kakizaki rats is caused by impaired Akt phosphorylation. Am J Physiol Cell Physiol 2009, 296:C327-C338.

56. Jude EB, Douglas JT, Anderson SG, Young MJ, Boulton AJ: Circulating cellular adhesion molecules ICAM-1, VCAM-1, P- and E-selectin in the prediction of cardiovascular disease in diabetes mellitus. Eur J Intern Med 2002, 13(3):185-189.

57. Soedamah-Muthu SS, Chaturvedi N, Schalkwijk CG, Stehouwer CD, Ebeling P, Fuller JH, EURODIAB Prospective Complications Study group: Soluble vascular cell adhesion molecule-1 and soluble E-selectin are associated with micro- and macrovascular complications in Type 1 diabetic patients. J Diabetes Complications 2006, 20(3):188-195.

58. Bates DO, Harper SJ: Regulation of vascular permeability by vascular endothelial growth factors. Vasc Pharmacol 2002, 39:225-237.

59. Simó R, Carrasco E, Garcia-Ramirez M, Hernandez C: Angiogenic and antiangiogenic factors in proliferative diabetic retinopathy. Curr Diabetes Rev 2006, 2:71-98

60. Yu XY, Chen HM, Liang JL, Lin QX, Tan HH, Fu YH, Liu XY, Shan ZX, Li XH, Yang HZ, Yang M, Li Y, Li SG: Hyperglycemic myocardial damage is mediated by proinflammatory cytokine: macrophage migration inhibitory factor. PLoS One 2011, 6(1):e16239.

61. Mano Y, Anzai T, Kaneko H, Nagatomo Y, Nagai T, Anzai A, Maekawa Y, Takahashi T, Meguro T, Yoshikawa T, Fukuda K: Overexpression of human $\mathrm{C}$-reactive protein exacerbates left ventricular remodeling in diabetic cardiomyopathy. Circ J 2011, 75(7):1717-1727.

62. Westermann D, Rutschow S, Van Linthout S, Linderer A, Bücker-Gärtner C, Sobirey M, Riad A, Pauschinger M, Schultheiss HP, Tschöpe C: Inhibition of p38 mitogen-activated protein kinase attenuates left ventricular dysfunction by mediating pro-inflammatory cardiac cytokine levels in a mouse model of diabetes mellitus. Diabetologia 2006, 49(10):2507-2513.

63. Yokoyama T, Nakano M, Bednarczyk JL, McIntyre BW, Entman ML, Mann DL: Tumor necrosis factor-aprovokes a hypertrophic growth response in adult cardiac myocytes. Circulation 1997, 95(5):1247-1252.

64. Sun M, Dawood F, Wen WH, Chen M, Dixon I, Kirshenbaum LA, Liu PP: Excessive tumor necrosis factor activation after infarction contributes to susceptibility of myocardial rupture and left ventricular dysfunction. Circulation 2004, 110(20):3221-3228.

65. Younce CW, Wang K, Kolattukudy PE: Hyperglycemia-induced cardiomyocyte death is mediated via MCP-1 production and induction of a novel zinc-finger protein MCPIP. Cardiovas Res 2010, 87:665-674.

doi:10.1186/1475-2840-12-44

Cite this article as: Nunes et al:: Early cardiac changes in a rat model of prediabetes: brain natriuretic peptide overexpression seems to be the best marker. Cardiovascular Diabetology 2013 12:44.

\section{Submit your next manuscript to BioMed Central and take full advantage of:}

- Convenient online submission

- Thorough peer review

- No space constraints or color figure charges

- Immediate publication on acceptance

- Inclusion in PubMed, CAS, Scopus and Google Scholar

- Research which is freely available for redistribution

Submit your manuscript at www.biomedcentral.com/submit
C Biomed Central 